\title{
Epileptic Capgras-Like Delusions in a Patient with Right Frontal Meningioma: Case Report
}

\author{
Eleonora Colombo ${ }^{a}$ b Stefano Messina ${ }^{a}$ Federico Verde $^{a}$ \\ Marco Locatelli ${ }^{b, c}$ Barbara Poletti ${ }^{a}$ Vincenzo Silani ${ }^{a}$ b Nicola Ticozzi ${ }^{a, b}$ \\ a Department of Neurology, Istituto Auxologico Italiano IRCCS, Milan, Italy; ${ }^{b}$ Department of \\ Pathophysiology and Transplantation, Università degli Studi di Milano, Milan, Italy; 'Division \\ of Neurosurgery, Fondazione IRCCS Ca' Granda, Ospedale Maggiore Policlinico, Milan, Italy
}

\section{Keywords}

Capgras syndrome $\cdot$ Epilepsy $\cdot$ Seizure $\cdot$ Meningioma

\begin{abstract}
Capgras syndrome is a condition characterized by the belief that a relative has been replaced by an almost identical imposter. The disorder has been reported in several neurological diseases. We describe the uncommon case of a transient Capgras syndrome manifesting as focal temporal seizures in a woman with a right frontal meningioma. Our patient represents an exceptional case of Capgras syndrome for several reasons, namely, the association with meningioma, very rarely reported before, the transient manifestation of symptoms, and, most importantly, the epileptic etiology of reduplicative paramnesias. Lastly, our report also confirms the importance of frontal and right hemisphere dysfunction in generating Capgras syndromelike delusions.
\end{abstract}

\section{Introduction}

Capgras syndrome is a condition characterized by the belief that a close relative has been replaced by an almost identical imposter. The disorder, originally described by Capgras and Roubal-Lachaux in 1923, has long been associated with psychiatric diseases, such as paranoid schizophrenia and schizoaffective disorder, but more recent reports also postulate an organic basis. Indeed, Capgras syndrome has been described in several neurological disorders, both neurodegenerative and nonneurodegenerative, such as cerebrovascular disease, head trauma, brain tumor, Alzheimer disease, and Lewy body disease [1]. In the context of epilepsy, 
the occurrence of Capgras syndrome has been documented mainly as a postictal or interictal manifestation. Here, we describe a case of focal seizures presenting as transient Capgras syndrome, which, based on the existing literature, represents an exceptional manifestation of this syndrome.

\section{Case Presentation}

An 83-year-old woman abruptly presented one morning with the belief that her husband had been replaced by a nearly identical substitute. She repeatedly asked him who he was and where she could find her real husband, to the point of showing around a picture of him and calling his work office for help. During that day, the patient was able to manage her daily activities as usual, she prepared lunch and dinner, but she seemed often confused. She believed that her dead father was still alive and looked for him, she did not recognize some objects in her home, and she thought that a third person lived with them. She did not experience hallucinations or prosopagnosia. These symptoms gradually disappeared during the following day, when she was able to correctly recognize her husband. Although she was initially unable to recall what happened, the patient regained her memories of that day in the following weeks.

Her past medical history revealed hypertension, rheumatic polymyalgia, hysterectomy to remove a fibroma, and an oncological history. Three years before, she was diagnosed with non-small cell lung carcinoma and pancreatic carcinoma with liver and diaphragmatic metastasis. She underwent radiation therapy first and then chemotherapy treatments with gemcitabine (8 cycles) with good response. The follow-up visits showed no disease progression. Since few months before the focal seizure, the patient also complained of right frontal headache.

The patient was evaluated at our outpatient neurology clinic 3 days after the event and hospitalized 1 week later. When the patient was admitted to our Department, the neurological examination was normal. Neuropsychologic evaluation revealed a mild cognitive

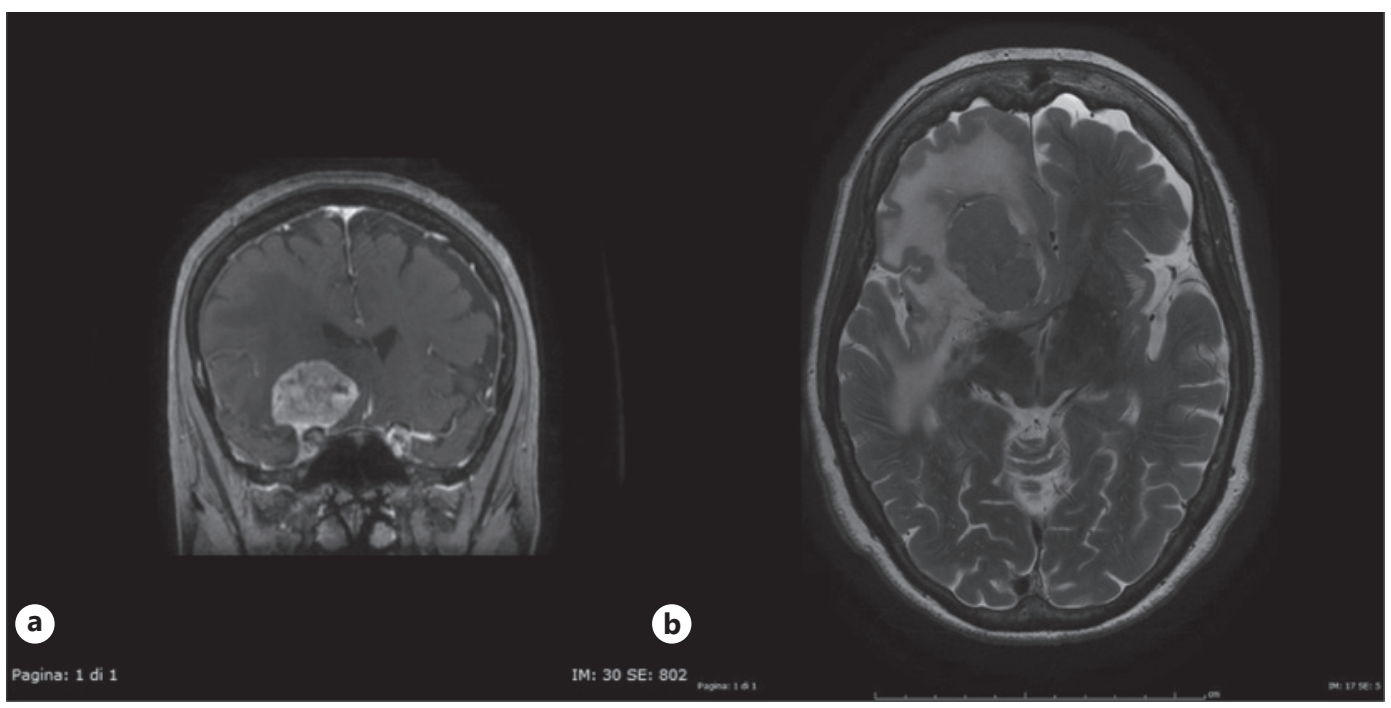

Fig. 1. Brain MRI. The exam showed a large right frontobasal extra-axial lesion (coronal T1-weighted image with gadolinium enhancement, a), with mild compressive effect on adjacent structures and marked perilesional edema (basal axial T2-weighted image, b). 
impairment characterized by selective frontal dysfunction with dysexecutive deficits and a minor involvement of language skills. The test showed no prosopagnosia or visuoperceptive impairment. A deflection of mood was also reported. EEG revealed focal theta activity on right frontal regions, and brain MRI showed the presence of a large right frontobasal meningioma with marked perilesional edema and mild compressive effect on adjacent structures (shown in Fig. 1). Surgical treatment was postponed because of age, comorbidities, and patient decision. During the hospitalization, the patient was started on levetiracetam $500 \mathrm{mg}$ b.i.d. and dexamethasone without seizure recurrence. In the following months after the discharge, the patient did not complain of confusion or delusional beliefs anymore, and regular followup visits (last in July 2020) showed a stable clinical condition.

\section{Discussion}

Capgras syndrome has been described in association with different brain tumors, such as pituitary tumor, glioma, and also with meningiomas. One of the first links between meningioma and Capgras syndrome was made by Kim [2] in 1991: he reported the case of an 80-year-old man with generalized seizures secondary to a right frontal meningioma developing initially transient, but eventually permanent, Capgras syndrome-like delusions [2]. Fennig et al. [3] in 1994 described a 43-year-old woman with a right frontal parasagittal meningioma that developed the delusion that her husband and children had been replaced by identical impostors. In this case, symptoms completely resolved after surgery, supporting the belief that the meningioma was directly involved in the syndrome [3]. However, Capgraslike delusions associated with brain tumors do not always disappear after surgery. In these patients, the surgical outcome likely depends on the nature of tumor (infiltrating vs. benign, noninfiltrating) and on the time of surgery, before strengthening of epileptogenic networks can occur.

The occurrence of Capgras syndrome has been documented in postictal delirium, such as in the Kim's case described above [2] and in other reports [4-6], but it was also described in the interictal period [7-9]. For example, Chawla and Virmani [7] reported the case of a young man suffering from temporal lobe epilepsy since he was 19 years old. When the patient was 26 years old, he also developed a concomitant delusional belief that his elder brother and father had been replaced by imposters. In this report, every patient's symptoms including Capgras delusion regressed after epileptic therapy adjustment. Instead, seizures manifesting with reduplicative paramnesia appear to be extremely rare, with only 1 case described in the literature (Table 1). Turtzo et al. [10] illustrated the case of an 87-year-old man presenting with both Capgras syndrome and severe unilateral spatial neglect during nonconvulsive status epilepticus; in this case, symptoms resolved after antiepileptic treatment [10].

In the context of an organic etiology of Capgras syndrome, many authors, such as Alexander et al. [11], have underlined the importance of bilateral frontal and right hemisphere dysfunction and tried to explain the underlying pathophysiological mechanism involved. Similarly, our case stresses the importance of nondominant hemispheric dysfunction in generating Capgras-like delusions, either epileptic or postictal. Right temporal lesions may impair visuospatial perception, facial recognition, and memory and could also be responsible for strange sensations of general familiarity or jamais vu. Furthermore, frontal deficits also prevent the reconciliation between present and past recollections of individuals or objects, and the disconnection between right frontal and limbic areas may cause a distorted sense of familiarity, thus generating reduplicative paramnesia [11].

\section{Karger'}


Table 1. Reported cases of Capgras syndrome-like delusions associated with epileptic syndromes

\begin{tabular}{|c|c|c|c|c|c|c|c|}
\hline Case & Age, yr & Gender & $\begin{array}{l}\text { Comorbid } \\
\text { diseases }\end{array}$ & $\begin{array}{l}\text { Seizure associated } \\
\text { with Capgras } \\
\text { syndrome }\end{array}$ & $\begin{array}{l}\text { Onset of } \\
\text { Capgras syndrome } \\
\text { delusions }\end{array}$ & $\begin{array}{l}\text { Duration of } \\
\text { Capgras syndrome } \\
\text { delusions }\end{array}$ & Ref. \\
\hline 1 & 80 & Male & $\begin{array}{l}\text { Right frontal } \\
\text { meningioma }\end{array}$ & Status epilepticus & Postictal & Permanent & [2] \\
\hline 2 & $\begin{array}{l}\text { Middle } \\
\text { age }\end{array}$ & Female & $\begin{array}{l}\text { Occipital lobe } \\
\text { epilepsy }\end{array}$ & $\begin{array}{l}\text { Focal, status } \\
\text { epilepticus }\end{array}$ & Postictal & Several days & [4] \\
\hline 3 & 55 & Female & $\begin{array}{l}\text { Generalized } \\
\text { epilepsy }\end{array}$ & Tonic-clonic & Interictal & Permanent & [8] \\
\hline 4 & 34 & Male & $\begin{array}{l}\text { Generalized } \\
\text { epilepsy }\end{array}$ & Tonic-clonic & Postictal & Some days & [5] \\
\hline 5 & 26 & Male & $\begin{array}{l}\text { Temporal } \\
\text { lobe epilepsy }\end{array}$ & $\begin{array}{l}\text { Tonic-clonic, } \\
\text { focal }\end{array}$ & Interictal & Weeks & [7] \\
\hline 6 & 87 & Male & None & $\begin{array}{l}\text { Nonconvulsive } \\
\text { status epilepticus }\end{array}$ & Ictal & Hours & [10] \\
\hline 7 & 27 & Male & $\begin{array}{l}\text { Nonspecified } \\
\text { epileptic } \\
\text { syndrome }\end{array}$ & $\begin{array}{l}\text { Focal to } \\
\text { tonic-clonic }\end{array}$ & Postictal & $\begin{array}{l}\text { Transient, } \\
\text { unspecified }\end{array}$ & [6] \\
\hline 8 & 40 & Female & $\begin{array}{l}\text { Nonspecified } \\
\text { epileptic } \\
\text { syndrome }\end{array}$ & $\begin{array}{l}\text { Focal to } \\
\text { tonic-clonic }\end{array}$ & Interictal & Months & [9] \\
\hline 9 & 83 & Female & $\begin{array}{l}\text { Right frontal } \\
\text { meningioma }\end{array}$ & Focal & Ictal & Hours & $\begin{array}{l}\text { This } \\
\text { report }\end{array}$ \\
\hline
\end{tabular}

In the context of epilepsy-associated Capgras syndrome, reduplicative paramnesia may appear because of ictal or postictal disinhibition of both the dominant and nondominant hemispheres, respectively, involved in recognition and in perceptual integration $[4,5]$. There is little information concerning the pathomechanisms of the 3 types (ictal, postictal, and interictal) of Capgras-like delusions. Ictal occurrence is extremely rare and, like in our patient, is the manifestation of a partial seizure involving right frontotemporal areas. According to the literature, it is possible to speculate that the postictal variant results from a transient disinhibition and dysfunction of the brain areas involved in the syndrome immediately after a seizure, similar to what is observed in postictal paralysis [5]. Conversely, the interictal variant may be explained by the strengthening of epileptogenic networks induced by repeated and/or untreated seizures. In fact, Kim [2] describes a patient with increasing persistence of delusions after each seizure to the point that Capgras syndrome eventually became permanent in the interictal phase. According to this hypothesis, repeated seizures could overtime alter interlobar signal transmission and interfere with frontal processing of visuospatial and affective memory.

Our patient represents an exceptional case of Capgras syndrome for several reasons, namely, the association with meningioma, very rarely reported before, the transient manifestation of symptoms, and, most importantly, the epileptic etiology of reduplicative paramnesias. Lastly, our report also confirms the importance of frontal and right hemisphere dysfunction in generating Capgras syndrome-like delusions.

\section{Karger'}




\section{Statement of Ethics}

Written informed consent was obtained from the patient for publication of this case report and any accompanying images.

\section{Conflict of Interest Statement}

The authors have no conflicts of interest to declare.

\section{Funding Sources}

This work was supported by the Italian Ministry of Health ("Ricerca corrente" to Istituto Auxologico Italiano IRCCS).

\section{Author Contributions}

Eleonora Colombo, Stefano Messina, Federico Verde, Marco Locatelli, and Nicola Ticozzi: patient evaluation and data acquisition. Eleonora Colombo and Nicola Ticozzi: drafting the manuscript. Marco Locatelli, Barbara Poletti, Vincenzo Silani, and Nicola Ticozzi: critical revision. Vincenzo Silani and Nicola Ticozzi: supervision.

\section{References}

1 Pandis C, Agrawal N, Poole NA. Capgras' delusion: a systematic review of 255 published cases. Psychopathology. 2019;52:161-73.

2 Kim E. A post-ictal variant of Capgras' syndrome in a patient with a frontal meningioma. A case report. Psychosomatics. 1991;32:448-51.

3 Fennig S, Naisberg-Fennig S, Bromet E. (Capgras' syndrome with right frontal meningioma). Harefuah. 1994; 126:320-67.367

4 Lilly B, Maynard E, Melvin K, Holroyd S. “Capgras” delusions involving belongings, not people, and evolving visual hallucinations associated with occipital lobe seizures. Case Rep Psychiatry. 2018;2018:1459869.

5 Joshi D, Koirala S, Lamichhane S, Paladugu A, Johal R, Lippmann S. Capgras syndrome in postictal delirium. Psychiatry. 2010;7:37-9.

6 Drake ME Jr. Postictal capgras syndrome. Clin Neurol Neurosurg. 1987;89(4):271-4

7 Chawla HM, Virmani V. Capgras phenomenon in a case of temporal lobe epilepsy. Folia Psychiatr Neurol Jpn. 1977;31:615-7.

8 Nejad AG, Toofani K. A variant of capgras syndrome with delusional conviction of inanimate doubles in a patient with grandmal epilepsy. Acta Neuropsychiatr. 2006;18:52-4.

9 Horikawa H, Monjji A, Sasaki M, Maekawa T, Onitsuka T, Nitazaka Y, et al. Different SPECT findings before and after Capgras' syndrome in interictal psychosis. Epilepsy Behav. 2006;9:189-92.

10 Turtzo LC, Kleinman JT, Llinas RH. Capgras syndrome and unilateral spatial neglect in nonconvulsive status epilepticus. Behav Neurol. 2008;20:61-4.

11 Alexander MP, Stuss DT, Benson DF. Capgras syndrome: a reduplicative phenomenon. Neurology. 1979;29: 334-9.

\section{Karger'k}

